\title{
An Approach to Rate Allocation in Multicast
}

\author{
Tudor Mihai Stoenescu \\ stoenesc@eecs.umich.edu \\ Mingyan Liu
mingyan@eecs.umich.edu \\ Demosthenis Teneketzis \\ teneketzis@eecs.umich.edu \\ Department of Electrical Engineering and Computer Science \\ University of Michigan \\ Ann Arbor, Michigan 48109-2122
}

\begin{abstract}
In multirate multicast different users in the same multicast group can receive services at different rates depending on their own requirements and the congestion level of the network. In this paper we present a general framework for addressing the optimal rate control problem in multirate multicast where the objective is the maximization of a social welfare function expressed by the sum of the users' utility functions.

We discover properties of optimal solutions of this problem. Based on these properties we describe a market mechanism that achieves an optimal solution and satis£es the informational constraints imposed by the decentralization of information in multicast service provisioning.
\end{abstract}

\section{INTRODUCTION}

Multicast provides an ef£cient method of transmitting data in real time applications from one source to many users. The source sends one copy of a message to its users and this copy is replicated only at the branching points of a multicast tree. Real time examples of such multicast applications are audio/video broadcasting, teleconferencing, distributed databases, £nancial information, electronic newspapers, weather maps and experimental data.

Conventional multicast studies the problem in which the rate received by all the users of the same multicast group is constant. The inherent problem with such a formulation is that a constant rate will overwhelm the slow receivers while starving the fast ones. Multirate transmissions can be used to address this problem by allowing a receiver to obtain data at a rate that satisfes its requirements. One way of achieving this is through hierarchical encoding of the transmission, in which a signal is encoded into multiple layers that can be incrementally combined to improve quality. These hierarchical encoding type of transmission schemes have been investigated both for audio and video transmissions over the Internet [3], [40] and over ATM networks [19]. Internet protocols for adding and dropping layers for hierarchical encoding type of transmissions are presented in [22] and [25].

Within the context of single rate and multirate multicast problems, studies have addressed issues of band- width/rate allocation $[4,9,12,14,27-32,35,41]$, routing $[5,6,26,34,42]$ and reliability $[7,10,15]$. Our work in this paper falls under the category of rate allocation for multirate multicast. Most of the literature on rate allocation is done via the notion of fairness $[4,9,27-32$, $35,41]$, specifcally, max-min fairness [2] and proportional fairness [17]. In particular, [32] develops a unifed framework for diverse fairness objectives via the notion of fair allocation of utilities. A more general approach to rate allocation is via utility maximization. Utility maximizing is more general because rate allocation with the fairness property are utility maximizing when the utility has a special form $[4,24,32,35]$. Although utility maximization has been extensively studied within the context of unicast rate allocation to achieve congestion control $[1,13,16,18,20,21,23,37,38]$, relatively fewer studies approached the multicast rate allocation problem via solving a general utility maximization formulation, with the notable examples being [12,14]. In [12] the authors propose a distributed and scalable algorithm for the problem, whose solution is based on dual methods. In [14] the authors derive a primal algorithm based on non-differential optimization methods. This algorithm is scalable and distributed and does not require prior knowledge of the utility functions.

In this paper we present a market-based approach to multicast service provisioning. This market-based approach has the following characteristics: (i) it is distinctly different from the approach in [12] and [14] because it adopts a hierarchical architecture (see Section IV) as opposed to the at architecture of [12] and [14], and because it employs a Tatônment process different from that of [12] and [14]. (ii) It satis£es the informational constraints that are present in network resource allocation problems and are discussed in Section IV of the paper. (iii) It achieves a solution that maximizes a social welfare function that is the sum of users' utility functions.

The major contribution of this paper is the development of a general framework for multicast service provisioning. Within this framework the key features of 
our approach are:

1) The determination of optimal link price sharing by the network users. The methodology for characterizing optimal price sharing is different from that in [12] and [14]. In [12] a dual approach to determining price sharing is used. Our approach is based on the primal problem. A primal problem approach is used in [14] but the solution obtained in [14] does not rely on optimal price sharing. The philosophy of our approach to characterizing optimal price sharing is also different from that of $[8,11]$ which is based on cooperative game theory.

2) The description of a market-based mechanism which achieves a welfare maximizing (i.e. aggregate utility maximizing) solution based on the properties of optimal link price sharing hierarchical architecture.

The paper is organized as follows. In Section II we formally present the centralized multirate multicast problem. In Section III we develop properties of the optimal solution of this problem. These properties in turn determine optimal price sharing along each multicast tree. In Section IV we describe and analyze a competitive market economy which leads to decentralized rate allocations that achieve a solution of the centralized multirate multicast problem. We discuss and critique our results in Section V, and conclude the paper in Section VI.

\section{The Multicast Problem}

In this section we present the model and the mathematical formulation of a network multicast problem.

\section{A. The model, terminology and notation}

Consider a network consisting of a set of $L$ unidirectional links, each link $l \in L$ having £nite capacity $c_{l}$. The network is used by a set $M$ of multicast groups. Each multicast group $m \in M$ is specifed by $\left\{s_{m}, R_{m}, L_{m}\right\}$, where $s_{m}$ is the unique source node, $R_{m}$ is the set of receiver nodes, and $L_{m}$ is the set of links used by the group. Since each multicast group is a tree, we are going to use the terms multicast group and multicast tree interchangeably.

We denote by $R \triangleq \cup_{m \in M} R_{m}$ the set of all receivers over all the multicast groups, and by $R_{l, m}$ the set of all the receivers of multicast group $m \in M$ using link $l \in L$. For each multicast tree $m \in M$ and each link $l \in L_{m}$ we de£ne by "upstream" on the multicast tree, all the links and nodes connecting $l$ to the source node. A link $l^{\prime} \in L_{m}$ is said to be "downstream" of a link $l \in L_{m}$ if link $l$ is upstream of link $l^{\prime}$. A link $l \in L_{m}$ is said to be the parent of a link $l^{\prime} \in L_{m}$ if $l$ is upstream of $l^{\prime}$ and there are no intermediate links between $l$ and $l^{\prime}$.

\section{B. The Optimization Problem}

We assume that we have a unique user connected to each receiver node $r \in R$. For each user we have a utility function $U_{r}\left(x_{r}\right)$, where $x_{r}$ is the rate at which $r$ receives data. This utility function can be interpreted both from the point of view of perceived quality of service received or the amount paid in order to receive the service. Since there is a unique user connected to each receiver node, we will use the same notation when we talk about the receiver nodes or the users connected to these nodes.

Assumption 1. The utility functions $U_{r}\left(x_{r}\right)$ are strictly concave, increasing and continuously differentiable.

Consider the following network multicast problem for the model of Section II-A

$$
\max _{x_{r}, r \in R} \sum_{r \in R} U_{r}\left(x_{r}\right)
$$

$\operatorname{Max} 1$

subject to the following constraints:

$$
\begin{gathered}
\sum_{m \in M} \max _{r \in R_{l, m}} x_{r} \leq c_{l}, \quad \forall l \in L \\
x_{r} \geq 0, \quad \forall r \in R
\end{gathered}
$$

The constraint (II.1) is also known as the capacity constraint. For this constraint to be satis£ed, on each link, the totality of the rates used by each multicast tree can not exceed the link capacity. The capacity constraint insures that for all the multicast trees, the rate on each branch of a tree is less than or equal to the rate on its parent branch.

Noting that the constraints (II.1) and (II.2) make the set of feasible solutions ( $x$ 's) compact, and since $U_{r}^{\prime} s$ are assumed to be continuous, Weierstrass's Theorem [36, p.823] guarantees the existence of a solution to Max 1.

\section{Properties of Optimal Solutions of PROBLEM MAX 1}

We state properties of an optimal solution to Problem Max 1. These properties provide guidelines for the development of a Market-based decentralized algorithm that satis£es the informational constraints imposed by the nature of the multicast problem (described in Section IV-A) and achieves the solution to Problem Max 1. We have developed the properties by examining the Primal problem of Max 1, and we have related the prices seen by each individual user at an optimal solution of Problem Max 1 and the shadow prices of each individual 
links. These properties are summarized below. Their proofs can be found in [39].

- The computation of the prices seen by the users of a multicast tree is independent of the computation of the prices seen by the users of a different tree.

- For every link $l$ whose rate is less than the rate delivered on its parent link, the sum of shadow prices on the links downstream of $l$ is equal to the sum of the the prices seen by the users downstream of link $l$.

- For each multicast tree and each link $l$, the shadow price on each link is split into shares among the users downstream of link $l$ demanding the maximal rate.

- The service price of every user $r$ is the sum of the shadow price shares incurred on the links upstream of user $r$.

\section{A Market Based Realization of the Solution of Problem MAX 1}

We describe an algorithm that converges to a solution of Problem Max 1, and satis£es the informational constraints imposed by the nature of the network multicast problem (these informational constraints are described at the beginning of Section IV-A). We proceed as follows: First we describe a competitive market economy consisting of resource providers, service providers, users and an auctioneer. Within the context of this market we specify two procedures, one used by the service provider and one by the auctioneer, which lead to an allocation that achieves a solution to Problem Max 1.

\section{A. Description of the Market}

The economy consists of the following four types of agents: resource providers, service providers, users and an auctioneer. The auctioneer, the resource provider and the service provider know the topology of the network and the resources available to the network, but have no a priori information about the number of users that will request services and the preferences (utility function) of each user. Each user knows its own preferences (utility function) but has no information about the number of other users requesting services or their preferences, or about the topology of the network and the resources available to the network. The resource providers, service providers and users are price takers. They act as if their behavior has no effect on the equilibrium prices reached by the market allocation process. In our market the resource traded at each link is the available communication rate (i.e. bandwidth or capacity). The rate at each link is sold as raw material to the service providers. The rate price at link $l \in L$ will be denoted by $\lambda_{l}$. The service providers buy rates on various links from the resource providers. Using these rates, they set up services and the corresponding prices for each unit of these services. Then, they sell these services to the users.

We make the following observations: Because of the informational constraints mentioned above, we have two markets, one between the resource providers and the service providers, and the other between the service providers and the users. The price taking assumption and the fact that we try to maximize the user utilities imply that: (i) neither the resource nor the service providers will attempt to make a proft; and (ii) the service prices are directly derived from the resource prices. Therefore, we can look at the two markets as being one market, and we brieay describe the components of this market.

1) Resource providers: We assume that resource providers own the resources at each link, and that there is no cost associated with the supply of the resources to the market. Let

$$
y(\lambda) \in \underset{y \in Y}{\operatorname{argmax}} \sum_{l \in L} \lambda_{l} y_{l}
$$

be the aggregate supply at price $\lambda \triangleq\left\{\lambda_{l}\right\}_{l \in L}$, with $Y \triangleq\left\{y \in \mathbb{R}^{|L|}: 0 \leq y_{l} \leq c_{l}, l \in L\right\}$. From the assumption that there is no cost associated with the supply of resources to the market, equation (IV.1) has the solution $y_{l}=c_{l}$ for all $l \in L$ and $\lambda \geq 0$.

2) Service providers: The service providers receive a price per unit of resource for each link of the network from the resource providers. Since resource providers are non-pro£t makers, the resource prices seen by the service providers is the same as the link prices $\lambda$ set by the auctioneer.

A major challenge in solving multicast problems through pricing is the determination of the set of user service prices from the set of link prices. The dif£culty stems from the fact that in order to specify user $r^{\prime} s$ $(r \in R)$ service price, one needs to determine the optimal price share on each of the links upstream of user $r$, while satisfying the informational constraints imposed by the nature of the network problem. In [39, Appendix A] we present a distributed algorithm which computes for each user $r$ the set of service prices $p(r, \lambda)$ from the set of link prices $\lambda$. The set of service prices have the property that they generate a demand which maximizes the sum of users' utilities along any multicast tree given a $£$ xed set of link prices $\lambda$.

3) Users: Users are price takers and request service from the service providers. For each user $r \in R_{m}$ of the multicast tree $m \in M$ a service provider announces 
a service price $p(r, \lambda)$. Based on its service price, each user determines its desired service rate by solving:

$$
x_{r}(p(r, \lambda)) \triangleq \underset{x}{\operatorname{argmax}}\left\{U_{r}(x)-p(r, \lambda) \times x\right\}
$$

4) Auctioneer: The role of the auctioneer is to regulate the prices of the resources, based on the aggregate excess demand vector $z(\lambda)$,

$$
z_{l}(\lambda) \triangleq \sum_{m \in M} \max _{r \in R_{l, m}} x_{r}(p(r, \lambda))-c_{l}
$$

at every link $l \in L$.

\section{B. The Market Mechanism}

We present a market mechanism, described by an algorithm, that proceeds iteratively as follows:

Step 1: The auctioneer announces prices $\lambda$ per unit of resource/rate at each link of the network. The multicast trees are £xed.

Step 2: Based on the auctioneer's announcement, the service provider computes the price per unit of rate for each user, and announces the prices to the users. This is accomplished by an algorithm described in [39, Appendix A].

Step 3: Based on the prices $p$ announced by the service provider, the users request services in the amount $x(p)$ satisfying (IV.2).

Step 4: Based on the service demand vector $x(p)$, the auctioneer computes, through (IV.3), the sign of the excess demand vector $z(\lambda)$.

Step 5: If $z_{l}(\lambda) \leq 0$, for all $l \in L$, then the process ends. Otherwise the auctioneer changes the prices per unit of rate $p$ according to Scarfs' algorithm [33], and the process is repeated from Step 2 on.

The above steps are pictorially shown in Figure 1. The fgure illustrates the fact that the algorithm contains two loops: an outer loop and an inner loop. The inner loop describes the iterative process used by the service provider to determine user service prices (hence user demands) for £xed link prices set by the auctioneer. The outer loop determines the iterative process used by the auctioneer to determine link prices based on excess demand. The two iterative procedures are presented in detail in [39].

\section{The Main Result}

The main result of this paper is summarized by the following theorem:

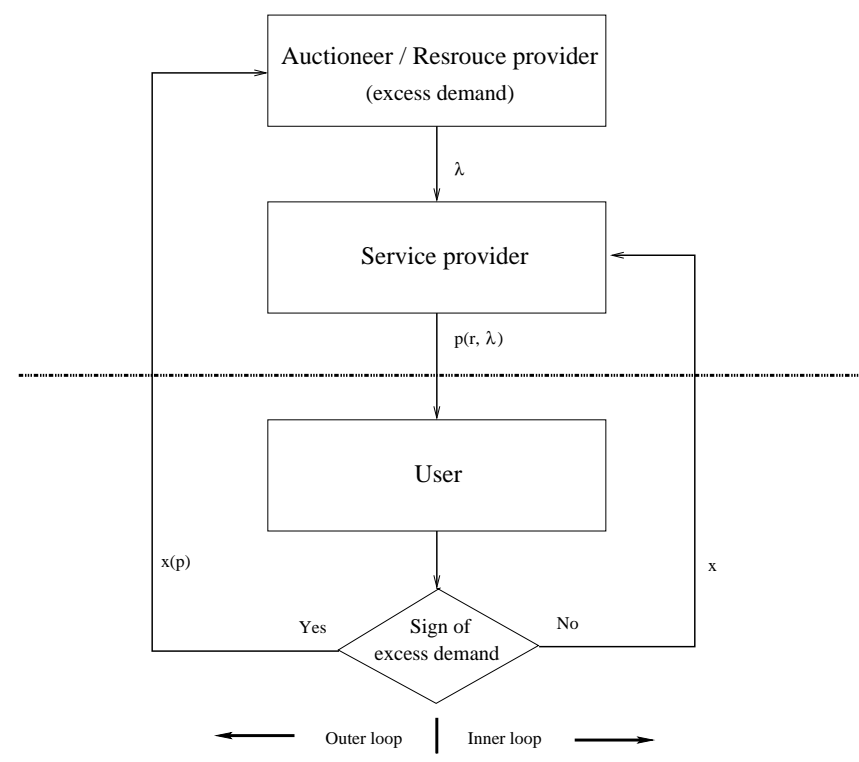

Fig. 1. Market mechanism.

Theorem IV.1. The decentralized allocation mechanism described in Section IV-B leads to an allocation that achieves an optimal solution to Problem Max 1.

The proof of Theorem IV.1 can be found in [39].

\section{CRitique, Discussion And Reflection}

The main features of the approach to the multirate multicast problem presented in this paper are the following:

(1) The agents are price takers in the markets in which they participate.

(2) There is no cost associated with the supply of network resources.

(3) The users' utility functions $U_{r}(x)$ are strictly concave, increasing and continuously differentiable.

(4) The objective of the resource allocation process is to maximize the total value of the network to its users.

We have presented extensive discussion and critique of features 1, 2 and 4 in $[37,38]$. The assumptions made on the users' utility functions are the typical ones appearing in analysis of economic optimization problems. Such assumptions are reasonable when we are dealing with goods that are desirable.

The issues associated with our approach to the multirate multicast rate allocation problem formulated in this paper are described in detail in [39]. In this document we address only the following: 
- The main difference in the setup of this problem as oppose to the problem addressed by $[37,38]$ is the computation of the service prices for each one of the users given a £xed link price per unit of rate. In the unicast problem the price seen by each user is equal to the sum of the link prices on the links connecting the user to the source. Since users share links in a multicast tree, the derivation of the service price for each user is much more complex. In Section III we presented properties of the optimal price sharing at the optimal solution of Max 1. This set of properties dictate how the set of given link prices are shared among the users. The algorithm described in [39, Appendix A] satisfes these properties, and under a set of optimal link prices it determines an optimal set of service prices. The users' demand based on these service prices generate a solution of Max 1.

- In [39] we developed a pricing mechanism based on Scarf's work [33] and proved that this mechanism converges to an optimal solution of Max 1. We note that if certain stronger constraints are set on the utility functions (twice continuously differentiable) then pricing mechanism that converges faster to an optimal solution of Max 1 can be developed.

\section{CONCLUSION}

In this paper we have presented an approach for optimal rate allocation in multirate multicast networks. We described a decentralized convergent iterative procedure that leads to a solution of a general rate allocation problem.

The contributions of this paper are:

1) The determination of optimal link price sharing by the network users.

2) The description of a market-based mechanism which achieves an aggregate utility maximizing (i.e. welfare maximizing) solution based on the properties of optimal link price sharing.

Acknowledgement: This research was supported in part by NSF Grant RCS-9979347 and by ONR Grant N00014-03-1-0232.

\section{REFERENCES}

[1] Y. Bartal, J. Byers, and D. Raz. Global optimization using local information with applications to aow control. In Proceedings of the $38^{t h}$ Ann. IEEE Symp. on Fundations of Computer Science (FOCS), Miami, FL, October 1997.

[2] D.P. Bertsekas and R. Gallagher. Data Networks. Prentice Hall, second edition, 1992.
[3] T. Bially, B. Gold, and S. Seneff. A technique for adaptive voice dow control in integrated packet networks. IEEE Transactions on Communications, 28(3):325-333, March 1980.

[4] S. Deb and R. Srikant. Congestion control for fair resource allocation in networks with multicast aows. In Proceedings of Congerence on Decision and Control CDC, Orlando, FL, December 2001.

[5] M. J. Donahoo, K. Calvert, and E. W. Zegura. Center selection and migration for wide-area multicast routing. Journal of High Speed Networks, 6(2), 1997.

[6] M. J. Donahoo and E. W. Zegura. Core migration for dynamic multicast routing. In ICCCN, Washington, DC, 1996.

[7] N.G. Duffeld, J. Horowitz, D. Towsley, W. Wei, and T. Friedman. Multicast-based loss inference with missing data. IEEE Journal on Selected Areas in Communications, 2002.

[8] E.J. Friedman. Optimization based characterizations of cost sharing methods. Departamental Working Paper at Rutgers University, Department of Economics, June 1999.

[9] E. Graves, R. Srikant, and D. Towsley. Decentralized computation of weighted max-min fair bandwidth allocation in networks with multicast xows. In Proceedings Tyrrhenian International Workshop on Digital Communications (IWDC), Taormina, Italy, 2001.

[10] R. Gupta and J. Walrand. Average bandwidth and delay for reliable multicast. In Proceedings IFIP Performance, Istanbul, Turkey, October 1999.

[11] S. Herzog, S. Shenker, and D. Estrin. Sharing the "cost" of multicast trees: An axiomatic analysis. IEEE/ACM Transactions on Networking, 5(6), December 1997.

[12] K. Kar, S. Sarkar, and L. Tassiulas. Optimization based rate control for multirate multicast sessions. In Proceedings of INFOCOM, Alaska, 2001.

[13] K. Kar, S. Sarkar, and L. Tassiulas. A simple rate control algorithm for maximizing total user utility. In Proceedings of INFOCOM, Alaska, 2001.

[14] K. Kar, S. Sarkar, and Leandros Tassiulas. A scalable low overhead rate control algorithm for multirate multicast sessions. IEEE Journal of Selected areas in Communication, 20(8):1541-1557, October 2002. Special issue in Network Support for Multicast Communications.

[15] S. Kasera, G. Hjalmtysson, D. Towsley, and J. Kurose. Scalable reliable multicast using mul- 
tiple multicast channels. IEEE/ACM Transactions on Networking, June 2000.

[16] F.P. Kelly. On tariffs, policing and admission control for multiservice networks. Operations Research Letters, 15:1-9, 1994.

[17] F.P. Kelly. Charging and rate control for elastic traf£c. European Transactions on Telecommunication, 8(1):33-37, 1997.

[18] F.P. Kelly, A.K. Maulloo, and D.K.H. Tan. Rate control for communication networks: shadow prices, proportional fairness and stability. Operational Research Society, 49:237-252, 1998.

[19] F. Kishino, K. Manabe, Y. Hayashi, and H.Yasuda. Variable bit-rate coding of video signals for ATM networks. IEEE Journal on Selected Areas In Communications, 7(5), June 1989.

[20] S. Kunniyur and R. Srikant. End to end congestion control schemes:Utility functions, random losses and ECN marks. In Proceedings of INFOCOM, March 2000.

[21] R. La and V. Anantharam. Charge-sensitive TCP and rate control on the internet. In Proceedings of INFOCOM, Tel Aviv, Israel, March 2000.

[22] X. Li, S. Paul, and M. H. Ammar. Layered video multicast with retransmission (LVMR):Evaluation of hierarchical rate control. In Proceedings of IEEE INFOCOM, San Francisco, CA, 1998.

[23] S. Low and D.E. Lapsley. Optimization aow control I: Basic algorithm and convergence. IEEE/ACM Transactions on Networking, 7(6), December 1999.

[24] L. Massoulie and J. Roberts. Bandwidth sharing: Objectives and algorithms. In Proceedings of Infocom 1999, New York, USA, March 1999.

[25] S. McCanne, V. Jacobson, and M. Vetterli. Receiver driven layered multicast. In Proceedings of ACM SIGCOMM, Stanford, CA, 1996.

[26] W. B. Park, H. L. Owen, and E. W. Zegura. Sonet/SDH multicast routing algorithms in symmetrical three-stage networks. In ICC, Seattle, WA, June 1995.

[27] D. Rubenstein, J. Kurose, and D. Towsley. The impact of multicast layering on network fairness. In Proceedings of ACM SIGCOMM, Cambridge, MA, 1999.

[28] S. Sarkar and L. Tassiulas. Fair allocation of resources in multirate multicast trees. In Proceedings of Globecom, 1999.

[29] S. Sarkar and L. Tassiulas. Distributed algorithms for computation of fair rates in multirate multicast trees. In Proceedings of INFOCOM, 2000.
[30] S. Sarkar and L. Tassiulas. Fair allocation of disrete bandwidth layers in multicast networks. In Proceedings of INFOCOM, Tel Aviv, Israel, 2000.

[31] S. Sarkar and L. Tassiulas. Back pressure based multicast scheduling for fair bandwidth allocation. In Proceedings of INFOCOM, Alaska, 2001.

[32] S. Sarkar and L. Tassiulas. Fair allocation of utilities in multirate multicast networks: A framework for unigying diverse fairness objective. IEEE Transactions on Automated Control, 47(6):931944, 2002.

[33] H. Scarf. The Computation of Economic Equilibria. Yale University Press, New Haven and London, 1973.

[34] J. K. Shapiro, J. Kurose, D. Towsley, and S. Zabele. Topology discovery service for router-assisted multicast transport. In Proceedings of OpenArch, 2002.

[35] J. K. Shapiro, D. Towsley, and J. Kurose. Optimization-based congestion control for multicast communications. In Proceedings of Networking, 2002.

[36] C.P. Simon and L. Blume. Mathematics for Economists. W. W. Norton, New York, 1994.

[37] T.M. Stoenescu and D. Teneketzis. A pricing methodology for resource allocation and routing in integrated-service networks with quality of service requirements. Mathematical Methods of Operations Research (MMOR), 56(2), 2002.

[38] P. Thomas, D. Tenektezis, and J. K. MacKieMason. A market - based approach to optimal resource allocation in integrated - services connection- oriented networks. Operations Research, 50(5), July-August 2002.

[39] T.Stoenescu, M. Liu, and D. Teneketzis. A pricing mechanism for optimal rate allocation in multicast service provisioning. Technical Report CGR 03-07, University of Michigan, March 2003.

[40] T. Turletti and J.C. Bolot. Issues with multicast video distribution in heterogeneous packet networks. Packet Video Workshop, 1994.

[41] H. Y. Tzeng and Siu K, Y. On max-min fair congestion for multicast abr service in atm. IEEE Journal on Selected Areas in Communication, 15(3), 1997.

[42] E. W. Zegura. Routing algorithms in multicast switching topologies. In Proceedings of Allerton Conference on Communication, Control and Computing, Monticello, IL, September 1993. 